\title{
TRAUMATISMOS CRANIANOS NA IDADE AVANÇADA
}

\author{
G. LAUSBERG * \\ D. KIRCHHOFF ** \\ J. A. REDONDO **
}

Os grandes avanços da gerontologia nos últimos anos não encontraram na neurocirurgia um éco tão grande quanto nas outras especialidades médicas. Em geral as contribuições encontradas na literatura médica se restringem aos aspectos diagnósticos e terapêuticos especiais dos tumores e insuficiências vasculares cerebrais nessa faixa etária (Cossel ${ }^{3}, K_{l o s s}{ }^{6}, \operatorname{Laux}^{7}, \mathrm{Pia}^{12}$ ). Quanto aos traumatismos cranianos, apesar do aumento de sua freqüência devido ao aumento dos acidentes automobilísticos nos últimos anos, poucas são as referências à problemática imposta pela idade avançada dos pacientes. Brun 2, em 1963, fez uma comunicação sobre 125 casos pré-senis e senis selecionados entre 1648 casos de traumatismo craniano, dos quais $282 \mathrm{em}$ pacientes com mais de 50 anos de idade, tendo estudado especialmente os aspectos médicos-legais. Do ponto de vista clínico-experimental só conhecemos os trabalhos de Walther ${ }^{19}$ (1960), sobre a comoção cerebral em pacientes idosos. Nos textos clássicos os traumatismos cranianos em pacientes idosos não são separados dos demais traumatismos cranianos; somente têm sido assinalados alguns aspectos clínicos e anátomo-patológicos, em estudos versando sobre o mecanismo da ação traumatisante (Hellenthal ${ }^{4}$, Isfort ${ }^{5}$, Loew ${ }^{9}$, Meixner ${ }^{10}$, Sjövall ${ }^{15}$, Strassmann ${ }^{16}$, Tönnis ${ }^{18}$ ).

M A T E R I A L

Dividimos nosso material, segundo orientação da escola de Tönnis, em traumatismos cranianos fechados (leves e graves), traumatismos cranianos abertos e hematomas intracranianos pós-traumáticos com caráter expansivo. O material consta de 2044 traumatismos de crânio, sendo 328 abertos e 1716 fechados (1043 leves e 673 graves); registramos 287 casos de hematomas intracranianos. Em pacientes com idade superior a 50 anos tivemos: 185 casos $(\mathbf{1 7 , 7} \%)$ de traumatismos cranianos fechados leves; 92 casos $(13,6 \%)$ de traumatismos cranianos fechados graves; 22 casos $(6,3 \%)$ de traumatismos cranianos abertos e 42 casos $(14,6 \%)$ com hematomas intracranianos pós-traumáticos.

Para evitar repetição no texto, usaremos as seguintes siglas para designar os diferentes grupos de casos: TCFL $=$ traumatismos cranianos fechados leves; TCFG = traumatismos cranianos fechados graves; TCA = traumatismos cranianos abertos; $\mathrm{HI}=$ hematomas intracranianos.

* Professor de Clínica Neurocirúrgica da Universidade de Giessen. (Diretor: Prof. H. W. Pia); ** Assistentes. 


\section{RESULTADOS COMENTADOS}

A relação entre TCFL (1043 casos), de um lado, e a soma de TCFG e TCA (1001 casos) de outro, se aproxima de 1:1 em todo o material. Nos pacientes idosos essa relação se aproxima de $2: 1$ a favor dos traumatismos leves (185 e 114 casos, respectivamente).

A mortalidade nos casos de TCFG foi de 4,3\% nos pacientes idosos e de $1 \%$ nos pacientes mais jovens. Mortalidade bem mais alta ocorreu nos casos de TCFG e TCA $(59,7 \%)$, como também nos HI $(85,7 \%)$. Esses dados são semelhantes aos registrados na literatura. Segundo Brun $^{2}$ a morbidade nos pacientes com mais de 50 anos de idade cai significativamente devido à menor exposição destes pacientes a acidentes de trabalho e de trânsito, o que foi confirmado pelo nosso trabalho. Após os 60 anos de idade a morbidade se eleva novamente, fato este que, na nossa opinião, está relacionado a uma reação psico-motora incerta própria dessa idade (típico exemplo disso são as quedas em escadarias com quase $20 \%$ nos nossos próprios casos).

Os registros feitos na literatura sobre a alterações da consciência imediatamente após um traumatismo de crânio em pacientes com idade avançada são contraditórias (Theato ${ }^{17}, 1949$; Brun ${ }^{2}$, 1963). Nestes pacientes, devido ao amplo espaço liquórico interno e externo e à espessa calota craniana, há uma proteção relativa do cérebro, o que explicaria a pouca freqüência de alterações da consciência no grupo dos TCFL. Por outro lado, se a ação física for suficiente para causar uma contusão cerebral, a freqüência e a persistência de alterações da consciência aumentam, principalmente após os 70 anos (Walther ${ }^{19}, 1960$ ). Sob este aspecto nosso material apresentou-se da seguinte forma: Dos 185 casos de TCFL, $127(68,6 \%)$ não apresentaram perda de consciência, ou ela foi inferior a 10 minutos; em 31,4\% dos casos o periodo de inconsciência foi maior. Nos 92 casos de TCFG não houve perda da consciência ou ela foi inferior a 10 minutos em 33,8\% dos casos; em $64,2 \%$ o periodo de inconsciência foi maior. Essa porcentagem nos TCA, com $50,0 \%$, se colocou entre os dois grupos precedentes. Alterações da consciência após intervalo livre foram assinaladas em 37 de nossos casos; só em 16 destes casos $(43,3 \%)$ pudemos, cirurgicamente ou mediante necrópsia, constatar um hematoma intracraniano. Do ponto de vista da morbidade e mortalidade pode-se dizer ainda que são diretamente proporcionais ao tempo de inconsciência e à idade.

A ocorrência de sinais neurológicos imediatamente após o traumatismo craniano, distribuiu-se entre os diversos grupos como segue: $23,7 \%$ nos casos de TCFL; 59,7\% nos casos de TCFG; 54,5\% nos casos de TCA; $69 \%$ nos casos de hematomas intracranianos, o que mostra uma franca dominância destes últimos.

A ocorrência de sinais neurológicos após um intervalo livre, nos quatro grupos e na mesma ordem, foi de $7,5 \%, 21,7 \%, 22,7 \%$ e $31,0 \%$, respectivamente. Dos 39 pacientes que apresentaram sinais neurológicos após um intervalo livre, somente $13(33,3 \%)$ tinham um hematoma intracraniano. Tanto a inconsciência como os sinais neurológicos após intervalo livre se mostram, 


\begin{tabular}{|c|c|c|c|c|}
\hline & \multicolumn{4}{|c|}{ Traumatismos cranianos } \\
\hline & \multicolumn{2}{|c|}{ fechado } & \multirow[t]{2}{*}{ aberto } & \multirow{2}{*}{$\begin{array}{l}\text { hematoma } \\
\text { intracraniano }\end{array}$} \\
\hline & leve & grave & & \\
\hline \multicolumn{5}{|l|}{ Pacientes conscientes ou } \\
\hline apenas ligeiramente confusos & 36 & 12 & $\mathbf{5}$ & 2 \\
\hline Inconsciência sem intervalo livre: & 149 & 80 & 17 & 40 \\
\hline menos que 10 minutos: & 91 & 19 & 6 & 1 \\
\hline mais que 10 minutos: & 54 & 29 & 3 & 5 \\
\hline mais que uma hora: & 3 & 17 & 7 & 18 \\
\hline mais que 24 horas: & 1 & 12 & 1 & 13 \\
\hline mais que uma semana: & - & 3 & 一 & 3 \\
\hline Inconsciência com intervalo livre: & 12 & 19 & 6 & 16 \\
\hline Inconsciência contínua até operação & & & & \\
\hline ou óbito & 8 & 54 & 11 & 26 \\
\hline
\end{tabular}

Tabela 1 - Modificações da consciência após traumatismos cranianos em pacientes com mais de 50 anos de idade.

nessa faixa etária, como maus indicadores da existência de um hematoma intracraniano, não cooperando para o diagnóstico diferencial. Além disso, a lesão de nervos cranianos é relativamente comum e parece estar relacionada ao grande número de fraturas da base do crânio nos traumatismos cranianos na idade avançada, sendo outros achados clínicos (hemiplegias, decerebração) iguais ao de grupo de pacientes mais jovens. Queremos, no entanto, sublinhar o grande número de crises epilépticas precoces $(3,1 \%)$ nestes pacientes, contrariamente à difundida opinião de estar diminuido o potencial epileptogênico na idade avançada.

\begin{tabular}{|c|c|c|c|c|}
\hline & & \multicolumn{2}{|c|}{ Traumatismos cranianos } & \multirow{3}{*}{$\begin{array}{c}\text { hematoma } \\
\text { intracraniano }\end{array}$} \\
\hline & \multicolumn{2}{|c|}{ fechado } & \multirow[t]{2}{*}{ aberto } & \\
\hline & leve & grave & & \\
\hline Sinais neurológicos primários & 44 & 55 & 12 & 29 \\
\hline Sinais neurológicos secundários & 14 & 20 & 5 & 13 \\
\hline Lesão de nervos cranianos & 30 & 41 & 15 & 31 \\
\hline Lesāo do $3 .^{\circ}$ par craniano (anisocoria) & 26 & 37 & 10 & 27 \\
\hline Hemiplegias & 19 & 68 & 17 & 42 \\
\hline Alteraçōes da pressão arterial & 12 & 24 & 3 & 22 \\
\hline Ataques epilépticos generalizados & 2 & 4 & 1 & - \\
\hline Ataques epilepticos focais & 一 & 3 & 4 & 3 \\
\hline Fratura da convexidade & 15 & 28 & 12 & 18 \\
\hline Fratura de base de crânio & 3 & 25 & 3 & 9 \\
\hline Fraturas mistas ou combinadas & 12 & 19 & 7 & 11 \\
\hline Decerebração & 1 & 23 & 8 & 21 \\
\hline
\end{tabular}

Tabela 2 - Aspectos clínicos após traumatismos cranianos em pacientes com mais de 50 anos de idade. 
Traumatismos cranianos nestes pacientes apresentaram as seguintes complicações: em 42 casos ocorreram hematomas intracranianos; em 61 casos ocorreram, adicionalmente ou somente, complicaçōes pulmonares, sendo esta última a complicação mais freqüente; complicações cardiacas ocorreram 16 vezes. Importante foi o comportamento da pressão arterial: em 139 casos $(46,6 \%)$ ela se apresentou fora de seus limites normais pré-traumáticos; 80 casos apresentaram hipotensão arterial; em 26 casos ocorreu hipertensão cuja causa foi, por 2 vezes $(84,5 \%)$, um hematoma intracraniano. Alternância de pressão arterial foi verificada em 33 pacientes.

A influência de fatores hemodinâmicos pode ser demonstrada no seguinte exemplo:

Um paciente com 62 anos de idade sofreu uma queda de $3 \mathrm{~m}$ de altura, ficando inconsciente por duas horas; quando visto em nossa clínica já contactuava, mas ainda estava confuso; algumas horas mais tarde apresentou-se novamente inconsciente, com reflexos exaltados e sinal de Babinsky à direita; o valor da pressão arterial caiu de $170 / 110 \mathrm{~mm} \mathrm{Hg}$ para $110 / 60 \mathrm{~mm} \mathrm{Hg}$ e, ao mesmo tempo, surgiu uma insuficiência respiratória relativa; radiologicamente foi encontrada uma fratura parietal do lado direito estendendo-se até a base do crânio; os exames complementares não mostravam indício de hematoma intracraniano; vinte e quatro horas depois, tendo a pressão arterial voltado aos valores iniciais e com a estabilização das condiçōes respiratórias, ocorreu significativa melhora do nível da consciência; alguns dias mais tarde foi dada a alta hospitalar estando o paciente em bom estado. Dez anos mais tarde, agora com 72 anos de idade, o paciente sofreu um acidente automobilistico com TCFL, ficando inconsciente por 10 minutos; ao ser internado o paciente estava desorientado e sonolento com midriase à esquerda, hemiparesia á direita e afasia, com pressão arterial de 210/140 mm Hg; radiologicamente foi verificada pequena fratura no parietal esquerdo; o ecoencefalograma mostrou deslocamento das estruturas medianas da esquerda para a direita. Após estabilizar as condições circulatórias e respiratórias, foi feita trepanação à esquerda com esvaziamento de amplo hematoma epidural. Na seqüência da evolução ocorreram, por duas vezes, ataques epilépticos generalizados apesar de medicação anticonvulsiva habitual e grave broncopneumonia que, com a ajuda de antibióticos, traqueotomia e aspiração bronco-traqueal, pôde ser curada. Durante toda essa evolução complicada foi possivel manter uma boa pressão parcial de oxigênio sangüineo e, além disso. manter a pressão arterial em níveis habituais ao paciente (170/110 $\mathrm{mm} \mathrm{Hg})$. Com essas medidas houve melhora acentuada nos sinais neurológicos inicialmente apresentados, podendo o paciente ser transferido para uma clínica de reabilitação.

Os 60 casos que foram autopsiados confirmaram os diagnósticos clínicos. Ao lado dos hematomas e contusões cerebrais estavam o edema cerebral e a embolia gordurosa. Nas complicações extra-cerebrais houve predominância das pulmonares. Dos 42 casos de hematomas intracranianos, 40 apresentaram contusões cerebrais. O número de lesões contusionais nos pacientes idosos com HI é, portanto, maior que em grupos de pacientes mais jovens.

Em nosso material os hematomas intracranianos ocorreram em 14,6\% dos casos o que é significativamente maior que em outros grupos etários, nos quais essa alteração abrange valores entre 3\% e 5\%. Além disso, 26 pacientes com HI apresentaram incosciência primária, 24 dos quais com inconsciência prolongada e 16 apresentaram um intervalo livre (38,2\%); 29 deles mostraram sinais neurológicos primários ao passo que apenas 13 apresentaram sinais neurológicos secundários. 
Alterações da pressão arterial e do pulso conseqüentes a alterações da pressão intracraniana foram assinaladas em 22 casos, pouco mais de $50 \%$. Neste grupo, ataques epilépticos ocorreram somente em 3 casos. Em 18 casos houve fratura da calota craniana, em 9 casos fratura de base de crânio, em 11 casos, fraturas da calota e da base. Quanto ao tipo de HI tivemos 15 hematomas epidurais, 18 hematomas subdurais, um hematoma intracerebral e 8 hematomas mistos. O melhor prognóstico nos casos com intervalos livres longos (mais de 12 horas), citado na literatura (Loew ${ }^{8}$ e Isfort $^{5}$ ) foi plenamente confirmado pelo nosso trabalho.

\begin{tabular}{|c|c|c|c|c|c|c|}
\hline & \multicolumn{6}{|c|}{ Complicações } \\
\hline & \multirow{2}{*}{$\begin{array}{l}\text { hematomas } \\
\text { intracranianos }\end{array}$} & \multirow[t]{2}{*}{ pulmonares } & \multirow[t]{2}{*}{ cardiacas } & \multicolumn{3}{|c|}{ hemodinâmicas } \\
\hline & & & & hipo & hiper & hiper-hipotensão \\
\hline TCFL & 2 & 14 & 10 & 43 & 10 & 27 \\
\hline TCFG & 35 & 38 & 6 & 21 & 16 & 5 \\
\hline TCA & 5 & 9 & $\longrightarrow$ & 16 & - & 1 \\
\hline
\end{tabular}

Tabela 3 - Algumas das complicações após traumatismos cranianos em pacientes com mais de 50 anos de idade.

\begin{tabular}{|c|c|c|c|c|}
\hline & \multicolumn{4}{|c|}{ Traumatismos cranianos } \\
\hline & \multicolumn{2}{|c|}{ fechado } & \multirow[t]{2}{*}{ aberto } & \multirow{2}{*}{$\begin{array}{c}\text { hematoma } \\
\text { intracraniano }\end{array}$} \\
\hline & leve & grave & & \\
\hline Edema cerebral & 6 & 17 & 4 & 9 \\
\hline Contusão cerebral & - & 44 & 10 & 33 \\
\hline Hematoma intracraniano & - & 28 & $\mathbf{5}$ & 33 \\
\hline Necrose & 2 & 5 & 一 & 1 \\
\hline Embolia gordurosa & 1 & 6 & 1 & 1 \\
\hline Sangramento tardio & - & - & - & 2 \\
\hline Processos cardíacos & 3 & 8 & - & 3 \\
\hline Pneumonia & 6 & 38 & 6 & 22 \\
\hline Aspiração pulmonar & 1 & 21 & 5 & 10 \\
\hline Embolia pulmonar & 2 & 6 & 1 & 2 \\
\hline Edema pulmonar & - & 9 & 3 & 4 \\
\hline Sangramentos do ap. digestivo & - & 8 & 4 & 5 \\
\hline Total de falecimentos & 8 & 54 & 13 & 37 \\
\hline Autópsias & 6 & 44 & 10 & 33 \\
\hline
\end{tabular}

Tabela 4 - Achados morfológicos e causa mortis num grupo de 299 pacientes com mais de 50 anos de idade que sofreram traumatismo craniano. 
Maior consideração de todos os problemas gerontológicos advém do fato de a vida média nos últimos 80 anos ter passado de 35,5 a quase 70 anos, isto é, ter-se duplicado. Assim, por exemplo, está sendo previsto que em 1980 a metade da população dos EUA contará com mais de 50 anos (Bodechtel ${ }^{1}$, 1965). Por isso o médico, qualquer que seja sua especialidade, terá de levar este fato em conta.

Nosso trabalho permite estabelecer as seguintes regras gerais a propósito dos principais problemas clínicos e terapêuticos nos casos de traumatismos cranianos na idade avançada: 1 - Apesar de curtos períodos de inconsciência e clinicamente considerados como TCFL, o grupo estudado apresenta maior mortalidade em comparação a grupos de pacientes mais jovens, o que deve ser atribuído à descompensação circulatória cerebral que nessa idade às vezes é irrecuperável; 2 - A inconsciência prolongada provocada por ações físicas fortes é maior no grupo de pacientes idosos e a mortalidade nesses casos já aumenta na idade pré-senil; 3 - São importantes as alterações da consciência, assim como sinais neurológicos que ocorram decois de intervalo livre, decorrentes de perturbações circulatórias gerais e locais, principalmente nos traumatismos graves, assim como de complicações pulmonares e cardíacas; 4 - A inconsciência primária, quase sempre devida a contusões, muitas vezes persiste com o desenvolvimento de um hematoma intracraniano; em apenas um terço destes casos a sintomatologia permite um diagnóstico de certeza; percentualmente o grupo dos hematomas intracranianos é maior do que em grupos de pacientes mais jovens; 5 - As lesões de nervos cranianos são mais freqüentes do que em grupos de pacientes mais jovens, devido ao maior número de fraturas da base de crânio; de outro lado chama a atenção o número relativamente alto de crises epilépticas pós-traumáticas; 6 - Pela dificuldade de estabelecer, somente pela sintomatologia, o diagnóstico precoce de hematomas intracranianos, os exames complementares (ecoencefalografia e angiografia) sempre devem ser feitos, possibilitando, assim, um tratamento cirúrgico imediato; $7-\mathrm{O}$ pronto reconhecimento das condições circulatórias do paciente e correspondente terapêutica são obrigatórios; 8 - Deve ser feita com urgência profilaxia contra estados hipoxêmicos secundários, isto é, de insuficiência respiratória, mediante traqueotomia (Nolte $\left.{ }^{11}, 196\right)$; 9 - Profilaxia contra complicações cardíacas; 10 - Mobilização precoce.

\section{R E S U M O}

Relato dos aspectos clínicos apresentados por 299 pacientes com mais de 50 anos de idade, que sofreram traumatismos cranianos. Em especial são abordadas as dificuldades no diagnóstico diferencial que os hematomas intracranianos apresentam nesse grupo etário (total de 42 casos). Complicações cerebrais e extra-cerebrais, isto é, extra-cranianas são discutidas, tendo-se especial cuidado nos casos com inconsciência secundária e/ou sinais neurológicos secundários. Comparação com grupos mais jovens é feita. São salientadas as medidas terapêuticas gerais que podem diminuir a alta quota de mortalidade. 
S U M M A R Y

\section{Head injuries in old age}

Report on the clinical aspects of 299 patients with head injury, all of whom were older than 50 years. The difficulties of differential diagnosis of intracranial hematomas within this group (42 cases) are discussed. The intracranial and extracranial complications are mentioned as causing a secundary disturbance of conscience. Therapeutical aspects that can diminish the presently still remarkable rate of mortality are discussed.

\section{Z USA M M EN A S U N G}

\section{Die Schädelhirnverletzungen im hohem Lebensalter}

Es wird berichtet über die klinischen Besonderheiten bei 299 Traumatikern eines neurochirurgischen Krankengutes, die das 50 Lebensjahr überschritten haben. Insbesondere werden die Differentialdiagnostischen Schwierigkeiten, die die intrakraniellen Haematome dieser Gruppe aufweisen (insgesant 42 Fälle) besprochen. Schliesslich wird auf die eingentlichen Zerebralen und extrazerebralen bzw. extrakraniellen Komplikationen unter Berücksichtigung häufigen Auftretens sekundärer Bewustseinstrübungen sowie neurologischer Ausfallserscheinungen hingeviesen. Therapeutische Gesichtspunkte die zu einer Senkung der heutigentags immer noch erheblichen Mortalitätsquote beitragen köntte, werden aufgezeigt.

REFERE NCIAS

1. BODECHTEL, G. - Das alternde Gehirn. Die Umschau in Wiss. und Tech. (Frankfurt a Mainz) 65:343, 1965.

2. BRUN, R. - Die Schädel und Hirnverletzungen. Huber, Bern-Stuttgart, 1963.

3. COSSEL, L. - Uber die Altersgebundenheit der Symptomatik der Hirntumoren. Zeitschrift für Ärztl. Fortbildung (Jena) 45:628, 1951.

4. HeLlenthaL, E. - Über das Zustandekommen, die Möglichkeit und die lokalisation der Contrecoup-Verletzungen des Gross und Kleinhirns. Deutsche Zeitchrift ges. gerichtliche Medizin. 21:231, 1933.

5. ISFORT, A. - Organische und funktionelle traumatische Gefässchäden im Röntgenbild. Hefte zur Unfallheilkunde Beiheft. 81:70, 1965.

6. KLOSS, K. - Hirntumoren höherer Altersstufen. Acta Neurochir. (Wien) 2:217, 1952.

7. LAUX, W. - Zur Diagnostik der Hirntumoren im höheren Lebensalter. Dtsch. med. wschr. 81:98, 1956.

8. LOEW, F. - Akute und subakute Störungen der zentralen Kreislaufregulation nach gedekten Hirnverletzungen. Zentralblatt Neurochirurgie (Leipzig) 9 Heft 2/3:128, 1949.

9. LOEW, F. - Die gedeckte Hirnschädigung als anatomiches und klinisches Problem. Zentralb. Neurochirurgie (Leipzig) 10:132, 1950.

10. MEIXNER, K. - Die Rolle der Gehirnershütterung bei den tödlichen Schädelverletzungen. Deutsche Zeitschrift ges. gerichtliche Medizin 6:105, 1926.

11. NOLTE, I. - Die Tracheotomie in der Neurochirurgie. Inaugural Dissertation, Giessen, 1965. 
12. Pia, H. W. - Altersprobleme in der Neurochirurgie. Acta Neurochir. (Wien) $5: 538,1957$.

13. PIA, H. W. - Fehler und Gefahren bei der Diagnose und Behandlung gedeckter Hirnverletzungen. Langenbecks Archiv für klinische Chirurgie. 298:110, 1961.

14. PIA, H. W. - Die Traumatischen Hirnblutungen des Kindesalters. Acta Neurochir. (Wien) 11:583, 1964.

15. SJOVALL, E. - Die Bedeutung der Altersveränderungen im Zentralnervensystem. Anatomischer Anzeiger. (Jena) 75:37, 1932.

16. STRASSMANN, G. - Uber Kopfverlezungen durch stumpfe Gewalt. Deutsche Zeitschrift ges. gerichtliche Medizin. 16:327, 1931.

17. THEATO, L. - Statistische Untersuchung über den Verlauf einfacher Commotionen bei 278 Unfallversicherten des Baugewerbes. Der Nervenarzt (Berlin) $13: 241,1949$.

18. TONNIS, W. - Die Chirurgie des Gehirns und seiner Häute. Die Chirurgie (Wien) 3:453, 1948 .

19. WALTER, K. - Die Commotio cerebri am alternden Hirn. Springer Verlag, Berlin-Göttingen, 1960.

Neurochirurgische Universitaets Klinik - Klinikstrasse 97 - 63 Giessen/LahnDeutschland. 